\title{
Philonsorbonne
}

9 | 2015

Année 2014-2015

\section{Penser « par la négative » : infortune et fortune d'une posture philosophique}

\author{
Manuel LEVAL-DUCHÉ
}

\section{Q OpenEdition}

Journals

Édition électronique

URL : https://journals.openedition.org/philonsorbonne/726

DOI : $10.4000 /$ philonsorbonne.726

ISSN : 2270-7336

Éditeur

Publications de la Sorbonne

Édition imprimée

Date de publication : 1 janvier 2015

Pagination : $51-70$

ISSN : 1255-183X

\section{Référence électronique}

Manuel LEVAL-DUCHÉ, «Penser «par la négative » : infortune et fortune d'une posture

philosophique », Philonsorbonne [En ligne], 9 | 2015, mis en ligne le 11 janvier 2015, consulté le 08 juin 2021. URL : http://journals.openedition.org/philonsorbonne/726 ; DOI : https://doi.org/10.4000/

philonsorbonne.726

(c) Tous droits réservés 


\title{
Penser «par la négative»: infortune et fortune d'une posture philosophique
}

\author{
Manuel LEVAL-DuCHÉ
}

Nous nous proposons, en premier lieu, de lire certains passages centraux du Visible et l'invisible de Merleau-Ponty à la lumière de l'analyse par John L. Austin du "doute ou de la question: "est-ce réel ou non ?" ». Nous pensons en effet qu'une telle lecture permet de clarifier certains attendus de ce texte de Merleau-Ponty, notamment autour de l'usage implicite d'un argument hérité de Bergson, et que cette lecture peut être versée au dossier d'un réalisme de Merleau-Ponty, ou du moins d'un « ton » réaliste de sa phénoménologie, solidaire d'une "subversion systématique de l'idéalisme transcendantal ${ }^{1} \gg$. Ce que nous voulons montrer d'abord, c'est donc la convergence forte, en particulier, de certaines analyses de Merleau-Ponty et d'Austin autour de la critique commune de certaines

1. Il s'agit par là, notamment, de « tester » une hypothèse de lecture allusivement proposée par J. Benoist, relative à l'existence d'un réalisme merleau-pontien, et selon laquelle l'auteur de la Phénoménologie de la perception contribue (par-delà les reculades et ambiguïtés qui traversent son œuvre) à construire un sens du réalisme qui, comme le dit Benoist de H. Putnam, «échappe aux pièges et aux apories métaphysiques qui lui sont trop souvent associés et qui font qu'aujourd'hui on peut parfois avoir de bonnes raisons d'éviter le mot "réalisme" " (J. Benoist, Sens et sensibilité. L'intentionalité en contexte, Paris, Éditions du Cerf, 2009, p. 261) : si Merleau-Ponty évite systématiquement le mot « réalisme » comme référent positif, "car il entend par "réalisme" réalisme métaphysique », " sa phénoménologie, en un certain sens, subversion systématique de l'idéalisme transcendantal, a une saveur typiquement réaliste. Le même Merleau-Ponty qui proclame le surmontement du "réalisme" [...] de la chose entendue comme en soi détachée de la perception et l'expérience humaine, est celui qui écrit aussi: "ce qui m'interdit de traiter ma perception comme un acte intellectuel, c'est qu'un acte intellectuel saisirait l'objet ou comme possible, ou comme nécessaire [modalisation propre à une perspective épistémologique], et qu'il est, dans la perception, 'réel' " (Le primat de la perception et ses conséquences philosophiques, Lagrasse, Verdier, 1996, p. 48) ». (J. Benoist, ibid., p. 261-262). 
«questions» canoniques mais fallacieuses que la philosophie se croit obligée de poser lorsqu'elle aborde le thème du réel. Mais nous souhaitons surtout en tirer quelques implications et conclusions plus générales quant au verdict que l'on peut rendre relativement à une posture philosophique consistant, pour le dire d'une formule lapidaire qui devrait s'éclairer progressivement, à "penser le positif par le négatif». Il s'agira alors d'élargir notre focale pour faire de Merleau-Ponty le représentant d'une inspiration théorique plus générale : nous voulons en effet prendre occasion de la discussion proposée dans la première partie de notre travail pour interroger la portée générale d'une certaine position philosophique, qui est en même temps un diagnostic critique sur une certaine façon, classique, d'envisager les problèmes en philosophie. Cette position, pour le dire de deux mots qu'il nous faudra développer, se nourrit d'une réception critique, voire d'un refus - non dénué parfois d'ambiguités et de reculades - du thème du transcendantal, compris de façon générale et schématique comme un mouvement de retrait, de désengagement, une "vue de côté » consistant par exemple à lire une capacité, un phénomène, voire la réalité dans son ensemble, d'un point de vue extérieur à eux, en se situant en retrait par rapport à eux, en un point où ils ne sont pas, depuis le lieu fictif ou hypothétique de leur absence - selon un suspens ou un détachement dont il s'agit de montrer qu'il est essentiellement artificiel, puisqu'il n'existerait pas, en vérité, un tel point de vue désengagé de l'agent par rapport à la réalité à partir duquel il serait possible de reconstruire les modalités de sa mise en contact avec celle-ci.

\section{La « ruse du métaphysicien »}

Mais commençons par rappeler l'analyse que propose Austin de l'usage ordinaire du mot « réel », dans un article traduit sous le titre « Autrui » dans les Écrits philosophiques, mais surtout au chapitre VII du Langage de la perception : cet usage est en effet un usage normatif ou polémique : il n'y a sens à dire de quelque chose que c'est réel que contre, pour récuser l'éventualité que ça ne le soit pas. La notion de réel intervient, dans son usage naturel, là où $i l$ y a à établir que c'est réel, là où contre l'éventualité que ça ne le soit pas, nous réaffirmons que c'est réel; autrement dit, la notion de réalité, comme la plupart des notions, est une notion polaire, qui intervient essentiellement, dans le langage ordinaire, pour disqualifier certaines choses, les qualifier, précisément, comme non réelles ou irréelles, là où il existe une (bonne) raison de croire qu'elles ne le sont pas. " Réel », comme d'ailleurs la formule «je sais » analysée par Wittgenstein, est une notion "spécialisée », c'est-à-dire polaire, polémique, oppositive. L'usage du concept de « réalité » est donc légitime là seulement où il est motivé et localisé. Plus précisément, analyse Austin, 
lorsqu'il s'agit de «réel», c'est l'usage négatif du mot qui «porte la culotte ». En d'autres termes, l'affirmation que quelque chose est réel, un réel tel ou tel, n'a un sens défini qu'à la lumière d'une manière spécifique dont ces $x$ pourraient être et pourraient avoir été non réels ${ }^{2}$.

«Réel ${ }^{3}$ reçoit donc tout son sens du contraste avec son opposé, et n'a d'emploi que dans des cas spéciaux, des circonstances polémiques particulières. Comme l'écrit Austin,

Le doute ou la question "mais est-il réel? » a toujours (doit avoir) un fondement particulier; il doit y avoir quelque « raison de suggérer » que ce n'est pas réel, au sens où il y a une façon spécifique, ou un nombre limité de façons spécifiques, de suggérer que telle expérience ou tel objet est peutêtre truqué ${ }^{4}$.

Et l'on pourrait ainsi distinguer, avec Austin, « entre défi épistémique (donner des raisons de répondre à la question "comment le savez-vous ?") et défi sceptique », « un défi sceptique [étant] un défi épistémique qui se pose en dehors des conditions authentiques et normales $»^{5}$. D'un côté, «les points [...] que pose, de façon tout à fait authentique et normale, la question "comment le savez-vous ?" "; de l'autre, "d'autres questions, que l'on considère habituellement comme plus importantes, $[\ldots]$ parfois posées sous la même rubrique, en particulier par les philosophes : ce sont les problèmes portant sur la "réalité" $[\ldots]{ }^{6}$. De la confusion de ces deux questions et de ces deux niveaux logiques (la question locale « comment le sais-tu ? » et le problème global de la "réalité ») naissent toutes les apories associées aux développements de la «querelle» métaphysique du réalisme.

2. J. L. Austin, Le langage de la perception, trad. fr. P. Gochet, revue par B. Ambroise, Paris, Vrin, 2007, p. 156. Pour un argument analogue, cf. L. Wittgenstein, Wittgenstein's Lectures, Cambridge 1932-1935, A. Ambrose (dir.), Blackwell, Oxford, 1979, à partir des notes de A. Ambrose et M. McDonald, trad. fr., Les cours de Cambridge 1932-1935, É. Rigal, TER, Mauvezin, 1988, p. 23.

3. Austin applique ici à «réel» une analyse déjà conquise à propos du couple « directement »/« indirectement ».

4. J. L. Austin, « Autrui », in Écrits philosophiques, trad. fr. L. Aubert et A.-L. Hacker, Paris, Seuil, 1994, p. 59.

5. C. Alsaleh, J. L. Austin et le problème du réalisme, thèse pour le doctorat de philosophie soutenue le 5 décembre 2003 à l'université de Picardie, Presses de l'A.N.R.T., p. 173. Cf. Austin, «Autrui », op. cit., p. 55 : "Jusqu'à maintenant, en me mettant au défi de répondre à la question “comment le savez-vous ?", vous n'êtes pas supposé avoir mis en doute mes références, vous avez cependant demandé à les connaître. Et vous n'avez pas non plus contesté les faits que j'avance (sur lesquels je m'appuie pour prouver que c'est un chardonneret), vous m'avez cependant demandé de les détailler. Voici un nouveau type de défi : la fiabilité de nos prétendues "références" et de nos prétendus "faits" est mise en question ».

6. J. L. Austin, « Autrui », op. cit., p. 57. 
Celles-ci consistent toujours à adosser le «problème» du réel à une «menace » littéralement «fantôme» et fictive, puisque le risque qu'on prétend faire courir relativement à la réalité en général n'a en fait pas de sens, en ce qu'on refuse de se donner un contexte précis (elle est censée être valable absolument).

Précisément, le geste du doute radical, ou, si l'on en vient déjà aux « cibles » de la réflexion de Merleau-Ponty, l'hypothèse de l'anéantissement du monde telle que présentée par exemple au $§ 49$ des Ideen I de Husserl, mais aussi finalement la réduction transcendantale elle-même ${ }^{7}$, ces gestes philosophiques consistent toujours à absolutiser une telle analyse, qui fait de l'usage de la notion de réel un usage nécessairement contextuel, appelé par un type particulier de problème : Husserl, comme d'autres avant lui, décontextualise, universalise cette analyse, en l'appliquant à un niveau où pourtant il paraît douteux qu'elle conserve un sens, en en faisant l'alpha et l'omega de notre rapport à la réalité en général, ce qui ouvre évidemment la possibilité d'un geste comme l'épochè et d'une hypothèse comme celle de l'anéantissement du monde. Or les remarques d'Austin ne consistent absolument pas à suggérer que nous ne saurions pas bien si les choses en général sont réelles ou pas : elles sont déflationnistes ; elles consistent bien plutôt à nous mettre devant l'évidence du fait qu'on voit mal ce que pourrait signifier de dire que sont réelles toutes sortes de choses par rapport à quoi la question ne se pose absolument pas; et même, à la limite, si l'on passe au registre métaphysique, ce que pourrait signifier de poser la question : «Le monde est-il réel ou pas ? » Là où, en effet, nous affirmons de quelque chose que c'est réel, ou là où nous disons, au contraire, que «ce n'est pas réel », en vérité, c'est toujours sur fond d'une sorte d' «être-de-plain-pied» au milieu des choses, selon lequel ces choses se présentent sous l'évidence de leur réalité. Et le point de l'analyse d'Austin est le suivant: si «bien évidemment " c'est réel, dans ce cas, quel sens pourrait-il y avoir à dire que ça l'est? Pour le dire en termes husserliens, la fonction exacte de l'analyse d'Austin semble être de nous suggérer qu'il n'y a tout simplement aucun sens à remettre en question l'attitude naturelle, puisque notre usage du mot « réel » est un usage qui fait fond sur cette attitude, qui la suppose toujours déjà.

Ce qui est visé à travers les «fausses questions » critiquées tant par Austin que (on va le voir) par Merleau-Ponty, c'est une analyse qui consiste donc à exporter le problème du type «telle chose que je distingue mal, ou dont j'ai des raisons locales de douter, est-elle réelle ? \ au point d'en faire un problème universel; et donc à adopter par là un préjugé épistémologique solidaire de l'argumentation sceptique traditionnelle et plus particulièrement de sa «prémisse centrale»: "Puisqu'il est toujours possible de douter de quelque chose, il est toujours possible de douter de tout»-la possibilité d'un doute ponctuel implique la possibilité d'un doute universel.

7. Cf. E. Husserl, Idées directrices pour une phénoménologie, I, trad. fr. P. Ricœur , Paris, Gallimard, 1950. 
Et Austin de définir ce qu'il appelle « la ruse du métaphysicien ${ }^{8} »$ :

[elle] consiste à demander : « est-ce une table réelle ? » (type d'objet dont on ne voit pas comment il pourrait être truqué), sans spécifier ni préciser ce qui ne va pas dans cette table, si bien que je ne sais absolument pas «comment prouver » que cette table est réelle. C'est la façon dont le mot « réel » est employé qui nous conduit à supposer que "réel» a une signification unique ( « le monde réel », « les objets matériels »), tout à fait mystérieuse et profonde. Au lieu de quoi nous devrions toujours insister pour spécifier ce qui s'oppose à « réel »- je devrais montrer « ce que ça n'est pas » pour montrer que quelque chose est « réel »?.

Autrement dit, et plus généralement, l'inférence fallacieuse est la suivante : de ce que je ne peux pas toujours savoir (pour prendre l'exemple proposé par Austin) si c'est un chardonneret ou pas, parce que parfois je n'y arrive pas (l'oiseau s'envole avant que j'aie pu contrôler de façon assez précise si c'en était bien un), certains ont tendance à conclure que je ne peux jamais le savoir ni le découvrir: transferts paradigmatiques du parfois (ou du quelquefois) au jamais (ou au toujours, mais c'est le même geste), du local au global, de la partie ou de l'objet au tout, du contextuel à l'universel, du polaire (ou polémique) à l'absolu, analogues dans leur structure, sont le cœur de l'erreur ou de la «ruse» qui est celle du « métaphysicien-prestidigitateur » d'Austin, lequel feint toujours de négliger que les cas de mise en doute sont toujours des cas particuliers ${ }^{10}$. Comme dit Merleau-Ponty dont les propos résonnent fortement avec ceux d'Austin,

8. Laquelle est aussi bien celle du «prestidigitateur», selon une équivalence que Austin formule expressément : "Les prestidigitateurs exploitent aussi cette ruse : "Un spectateur aura-t-il l'amabilité de s'assurer qu'il s'agit bien là d'un chapeau tout à fait ordinaire ?" Ce qui nous déconcerte et nous met mal à l'aise. D'un air penaud, nous disons qu'il semble en effet normal, tout en ayant conscience que nous n'avons pas la moindre idée de ce à quoi nous devons faire attention » (« Autrui », op. cit., p. 59).

9. Ibid., p. 59.

10. Cf. ibid., p. 59. On pourrait mettre en perspective cette « ruse» du métaphysicien en la rapprochant d'un geste rhétorique précis et d'une "question» à fois très célèbre et parodiquement paradigmatique de ce genre de fausses questions sur lesquelles prospère une certaine démarche philosophique - à savoir la question de Hamlet, To be or not to be, telle qu'elle est analysée par le linguiste Francis Goyet (nous suivons sur ce point V. Descombes, Les embarras de l'identité, Paris, Gallimard, 2013, p. 125 et suiv.). Goyet souligne en effet que Hamlet a appris la rhétorique en se livrant aux exercices classiques de cette discipline, parmi lesquels il en est un qui consiste à passer, justement, de la quaestio finita à la quaestio infinita. Par exemple, dans la progression des exercices scolaires, on pourra commencer par cette question finie limitée à un cas particulier: Caton doit-il se marier? Ensuite, on apprendra à traiter la question illimitée, infinie, correspondante en généralisant: L'homme doit-il se marier? Enfin, on en viendra à défendre la thèse ou l'antithèse de la question : Vaut-il mieux vivre marié ou non marié ? - Goyet considère alors la question posée par Hamlet : Être ou ne pas être? Hamlet se montre bon élève lorsqu'il change sa question personnelle, donc finie, en une question infinie : il ne s'agit plus seulement de lui, mais de tout être humain. Mais Hamlet ne s'en tient pas là, et il procède à une nouvelle généralisation de la question puisqu'il ne fait suivre le verbe « être » d'aucun attribut. Dès lors, tout se passe 
Que quelquefois les contrôles deviennent nécessaires et aboutissent à des jugements de réalité qui rectifient l'expérience naïve ne prouve pas que des jugements de cette sorte soient à l'origine de cette distinction, ou la constituent, et ne nous dispense donc pas de la comprendre pour elle-même ${ }^{11}$.

En effet, une question ne se pose, une description ou une qualification n'est appropriée, que si une alternative est possible; et l'usage du mot « réel », selon l'analyse d'Austin, n'est légitime qu'à vouloir distinguer ce qui est réel de ce qui ne l'est pas. Le problème est que l'usage qu'en font les philosophes critiqués par Austin (en l'occurrence Ayer) n'admet généralement pas ce contraste, ou du moins ne permet pas un contraste intelligible. La perception est « indirecte», nous disent-ils - mais que serait une perception " directe » ? Et, de même, quel est ce "rien » ou ce « néant » qu'on oppose à la réalité pour en penser le concept? Le cas contrastant n'ayant pas de sens, le contraste posé n'en a pas non plus ${ }^{12}$. Dès lors, les philosophes ne qualifient rien en disant de la perception qu'elle est indirecte, ou en construisant un concept "polémique» de la réalité dans son ensemble. Et les apories liées au problème métaphysique du réalisme sont en vérité fonction d'une opération théorique spécifique, que l'on peut qualifier comme décontextualisation, ou prétention illégitime à une généralité absolue ou « acontextuelle».

comme si la question était doublement infinie. Elle est « infinie » une première fois dans le sens des théoriciens de la rhétorique, c'est-à-dire qu'elle a un caractère général. Mais elle est de nouveau infinie au sens d'inachevée, car il y a maintenant un blanc dans la phrase interrogative. Par une première illimitation de l'interrogation, nous passons de « Caton doit-il faire ce qu'il faut pour être marié ? » à «Un homme, quel qu'il soit, doit-il faire ce qu'il faut pour être marié ? ». Ensuite, une seconde illimitation nous fait passer, de là, à «Un homme, quel qu'il soit, doit-il faire ce qu'il faut pour être... ? » Et cela revient à demander s'il vaut mieux pour quelqu'un être quelque chose, que de ne pas être quelque chose, et, finalement, par une nouvelle infinitation, être que de ne pas être. - Et il est bien clair que, si c'est là le sens de la question posée par Hamlet, cette question n'aura pas de réponse tant qu'on ne rétablira pas un attribut. Et c'est en fait exactement ce genre de généralisation acontextuelle propre à certaines questions philosophiques que j'ai en vue (cf. F. Goyet, Le sublime du lieu commun, Paris, Honoré Champion, 1993, p. 568-570).

11. M. Merleau-Ponty, Le visible et l'invisible, in CEuvres, éd. établie et préfacée par C. Lefort, Paris, Gallimard, « Quarto », 2010, p. 1673.

12. C'est exactement le principe de la critique wittgensteinienne du doute radical : il ne rime à rien, parce que si quelqu'un doutait par exemple de la réalité des objets physiques, « comment son doute se manifesterait-il en pratique ? Et ne pourrions-nous pas le laisser tranquillement douter, étant donné que cela ne fait en vérité aucune différence ? » (L. Wittgenstein, De la certitude, trad. fr. D. Moyal-Sharrock, Paris, Gallimard, 2006, 120., p. 47-48), et qu'on n'imagine même pas quelle différence cela pourrait faire ? Ce type de croyance " originaire » est en fait une croyance sans antithèse, pour laquelle aucun cas contrastant ne peut être posé : «Qu'est-ce que cela voudrait dire que de douter maintenant que j'aie deux mains ? Pourquoi ne puis-je absolument pas me le représenter? Qu'est-ce que je croirais si je ne croyais pas cela ? Je n'ai pas encore du tout de système dans lequel il pourrait y avoir ce doute » (Ibid., 247., p. 77). 


\section{L'argument de Bergson : la possibilité d'un doute (d'une négation) ponctuel implique-t-elle la possibilité d'un doute (d'une négation) universel ?}

Et là où le «quelque chose » qui s'oppose à « réel» n'est autre que le «néant » (c'est-à-dire lorsqu'on généralise l'« argument de l'illusion »), alors, selon un argument bergsonien dont il est intéressant de trouver quelque écho dans cette analyse d'Austin, il n'y a tout simplement aucun sens à poser la question de la réalité, faute d'un espace logique pertinent pour cette fin. Il y a là une inconséquence propre à ce qu'on pourrait appeler toute conception de la réalité comme « non-néant» ou « non-rien ».

Merleau-Ponty utilise en effet implicitement ${ }^{13}$, notamment dans le chapitre du Visible et l'invisible intitulé «Interrogation et intuition», un argument hérité de Bergson pour attaquer la possibilité même de l'épochè telle que définie par Husserl: le présupposé ultime d'un geste tel que la réduction phénoménologique, comme le montre Merleau-Ponty, c'est la détermination du sens d'être de la réalité à partir de la position préjudicielle du néant :

À partir des choses prises dans leur sens natif de noyaux identifiables, mais sans aucune puissance propre, on ne parvient à la chose objet, à l'En-soi, à la chose identique à elle-même, qu'en imposant à l'expérience un dilemme abstrait qu'elle ignore [...]. C'est en opposant à l'expérience des choses le fantôme d'une autre expérience qui n'en comporterait pas, qu'on l'oblige à dire plus qu'elle ne disait. [...] La chose ainsi définie, ce n'est pas la chose de notre expérience, c'est l'image qu'on en obtient en la projetant dans un univers où l'expérience ne se nouerait sur rien, où le spectateur se détournerait du spectacle, bref, en la confrontant avec la possibilité du néant. [...] Or, pouvons-nous penser l'expérience que nous avons en la profilant sur la possibilité du néant? L'expérience de la chose et du monde n'est-elle pas précisément le fond dont nous avons besoin pour penser de quelque façon que ce soit le néant ? $^{14}$

C'est parce que « la réalité » (ou le « monde »: le « réel » en position de substantif cette fois, et non plus d'adjectif) est abordée sur fond de néant et est déterminée par confrontation à la possibilité du non-être ; c'est parce qu'on confère au réel le sens essentiel de "non-néant» (geste dont on pourrait montrer qu'il est déjà présent, par exemple, dans les « Anticipations de la perception » de la Critique de la raison pure - mais on va voir que la référence critique à Kant doit être nuancée), que le réel est défini comme pur objet, et donc peut se voir « suspendu » sur le modèle de la suspension

13. Mais cette inspiration bergsonienne est explicitée par Renaud Barbaras : cf. R. Barbaras, Le tournant de l'expérience. Recherches sur la philosophie de Merleau-Ponty, Paris, Vrin, 1998, p. 70 et suiv.

14. Le visible et l'invisible, op. cit. p. 1786. 
d'un jugement local. Dès lors se trouve ouverte la possibilité, contestée par Merleau-Ponty, de la constitution du Lebenswelt («monde de la vie ») dans la subjectivité absolue, c'est-à-dire rien moins que le tournant idéaliste-transcendantal de la phénoménologie husserlienne.

Et en effet, selon l'analyse proposée par Merleau-Ponty, le geste fondamental dont procède ce qu'il appelle la "pensée objective » et, partant, la méconnaissance et le recouvrement du monde, se résume dans une certaine configuration de la question de la réalité, laquelle tombe bien sous le coup de la critique bergsonienne de la métaphysique. Cette critique est thématisée dans La Pensée et le mouvant ${ }^{15}$ et dans le quatrième chapitre de L'évolution créatrice; son principe irrigue, même malgré eux, les analyses tant d'Austin que de Merleau-Ponty.

Elle se cristallise dans l'analyse de l'usage du principe de raison suffisante, qui consiste (selon un mouvement naturel de la pensée) à demander pourquoi quelque chose existe plutôt que rien, c'est-à-dire à comprendre la réalité comme le produit d'une conquête sur le néant : « Je me dis qu'il pourrait, qu'il devrait même ne rien y avoir, et je m'étonne alors qu'il y ait quelque chose. Ou bien je me représente toute réalité comme étendue sur le néant, ainsi que sur un tapis : le néant était d'abord et l'être est venu par surcroît. » Certes, « l'être a pu se trouver toujours là : le néant, qui est rempli et comme bouché par lui, ne lui en préexiste pas moins, sinon en fait, du moins en droit ${ }^{16}$. Or c'est la position en pensée de ce «rien» (entendu comme néant de toute chose) qui pose d'emblée problème, en tant qu'elle viole les conditions de signification de ce qu'on peut désigner ainsi. On pourrait dire, en effet, en s'inspirant de Bergson, que ce rien, en se donnant comme la négation de toute chose, et non pas de telle ou telle chose, viole les conditions de sens de la négation. Au lieu de nier un étant déterminé, on nie l'étant comme tel, le « réel» comme tel, en sorte de constituer un néant radical, et l'on présente ainsi une alternative entre l'être et son contraire, sur le modèle de l'alternative entre telle chose et son absence. La question "Pourquoi y a-t-il quelque chose plutôt que rien ?» apparaît alors comme la simple généralisation de la question «Pourquoi y a-t-il telle chose plutôt que pas ? ». Or il paraît licite d'objecter à cela que nous n'avons aucune idée de ce que signifie une négation frappant toute chose, et que partant l'alternative que présente la «question » analysée par Bergson est fallacieuse : le seul usage que nous puissions comprendre de la négation est en effet celui qui se limite à une négation déterminée, c'est-àdire portant sur telle ou telle entité et non sur «la réalité » dans son ensemble. Autrement dit, la négation radicale n'est pas la généralisation légitime de la négation usuelle, mais une négation essentiellement autre, dont rien ne permet d'assurer qu'elle ait encore un sens.

15. Cf. H. Bergson, La Pensée et le mouvant, Paris, PUF, 1990, p. 105-108.

16. H. Bergson, L'évolution créatrice, in Euvres, éd. A. Robinet, Paris, PUF, 1991, p. 728. 
L'idée est donc que la question dite «canonique » fait un usage radical de la négation qui n'est pas simplement l'extension maximale de sa portée ordinaire, puisqu'entre l'extension de la négation à presque toute chose, ou à toujours davantage de choses (son extension indéfinie, toujours pensable), et son extension à toute chose sans exception, donc au Réel conçu comme Objet de tous les objets, il y a un saut qualitatif (comme de l'indéfini à l'infini actuel), qui nous fait passer de la négation d'un étant paraphrasable par la variation d'un autre étant à une négation qui n'est plus paraphrasable de cette façon, et donc qui ne correspond à aucun usage compréhensible du terme en question ${ }^{17}$. Ce sont bien là deux négations et deux « néants » de natures différentes, et non une seule négation, un seul «néant», dont la portée varierait de manière continue. Comme l'écrit Merleau-Ponty,

L'interrogation philosophique n'irait [...] pas au bout d'elle-même si elle se bornait à généraliser le doute, la question commune du an $\mathrm{sit}^{18}$, « est-ce que cela est », à les étendre au monde ou à l'Être, et se définissait comme doute, non-savoir ou non-croyance. Ce n'est pas si simple. En s'étendant à tout, la question commune change de sens ${ }^{19}$.

\section{Merleau-Ponty et la critique du «problème ontologique » : « on ne fait pas surgir l'être à partir du néant »}

\section{D'où qu'il puisse conclure :}

Le célèbre problème ontologique, le "pourquoi y a-t-il quelque chose plutôt que rien » disparaît avec l'alternative : il n'y a pas quelque chose plutôt que rien, le rien ne saurait prendre la place du quelque chose ou de l'être : le néant inexiste (au sens négatif) et l'être est, et l'exact ajustement de l'un sur l'autre ne laisse plus place à une question ${ }^{20}$.

Et de même que, selon l'analyse menée par Austin, il n'est d'emploi légitime que local pour le mot «réel», de même et corrélativement Merleau-Ponty peut dire qu'il n'est de «non-êtres» que «relatifs et localisés ${ }^{21}$. Le mouvement de pensée, naturel mais spécieux, qu'il faut interrompre, est donc le suivant: "l'Être est négation de la négation, il a une infrastructure de Néant, il est un attribut de la connaissance $\gg{ }^{22}$ Autrement dit, et encore une fois,

17. On suppose ici qu'un usage absolument non «polémique » de la négation, pour reprendre une catégorie élaborée par Reinach, viole les conditions de sens de ladite négation.

18. « Est-ce que cela est », par différence avec le quid sit, « ce que cela est ».

19. Le visible et l'invisible, op. cit., p. 1734-1735.

20. Ibid., p. 1696.

21. Ibid., p. 1697.

22. Ibid., p. 1698. 
Notre point de départ ne sera pas : l'être est, le néant n'est pas - et pas même : il n'y a que de l'être, formule d'une pensée totalisante, d'une pensée de survol - mais : il y a être, il y a monde, il y a quelque chose [...] On ne fait pas surgir l'être à partir du néant, ex nihilo, on part d'un relief ontologique où l'on ne peut jamais dire que le fond ne soit rien ${ }^{23}$.

Douter, biffer, nier, mettre en suspens même, n'ont donc de sens que local, sur fond d'une " basse continue » (" il y a quelque chose ») tacite, implicite, ininterrogée car indubitable ; généraliser ces opérations revient toujours à «jet[er] sur [le monde] l'ombre d'une inexistence possible», et donc à se tromper de problème, à poser " en face du monde » la mauvaise question: "elle n'est pas de savoir si le monde est vraiment ou s'il n'est qu'un rêve bien réglé [le "rêve cohérent» des Méditations cartésiennes] $[\ldots]$ Nous ne nous demandons pas si le monde existe, nous nous demandons ce qu'est pour lui qu'exister. ${ }^{24}$ »

On mesure donc la dette de Merleau-Ponty vis-à-vis de cette figure argumentative élaborée par Bergson ${ }^{25}$, dont l'esprit traverse le texte du Visible et l'invisible. Il s'agit toujours de jouer ce geste critique contre une certaine thématisation de la question de la réalité, qui revient à "prendre une assurance contre le doute, dont les primes sont plus onéreuses que la perte dont elle doit nous dédommager ${ }^{26}$ ". Ou encore : " C'est imposer à l'innocent la preuve de sa non-culpabilité ${ }^{27} \gg$.

23. Ibid., p. 1716-1717. Il faudrait citer l'ensemble du passage, et notamment la suite des phrases que nous reprenons : «Ce qui est premier, ce n'est pas l'être plein et positif sur le fond de néant, c'est un champ d'apparences, dont chacune, prise à l'écart, éclatera peut-être, ou sera biffée, dans la suite (c'est la part du néant), mais dont je sais seulement qu'elle sera remplacée par une autre qui sera la vérité de la première, parce qu'il y a monde, parce qu'il y a quelque chose, un monde, un quelque chose, qui n'ont pas, pour être, à annuler d'abord le rien $»$.

24. Ibid., p. 1723-1724.

25. Mais il serait intéressant, pour spécifier la nature exacte de la critique de la « fausse question » métaphysique, et pour montrer que cette critique relève d'un geste finalement assez partagé, de convoquer un autre argumentaire, qui consonne sur bien des points avec celui de Bergson: l'analyse syntaxique, par Kasimir Twardowski, de la représentation "rien» et de l'opération d'" infinitation » (c'est-à-dire la négation d'un nom) au début de «Sur la théorie du contenu et de l'objet des représentations » (in Sur les objets intentionnels (1893-1901), E. Husserl et K. Twardowski, trad. fr. J. English, Paris, Vrin, 1993) - avec l'idée, pour le dire en quelques mots, qu'il n'y a pas d'infinitation légitime du mot «quelque chose », et donc aucune dénotation et aucune signification pour le pseudo-nom « rien ».

26. Le visible et l'invisible, op. cit. p. 1670.

27. Ibid. On trouverait aisément, dans l'œuvre du dernier Wittgenstein, l'idée analogue, exprimée selon la même métaphore juridique quoique formulée dans un contexte fort différent, selon laquelle il est illégitime de traiter la réalité comme adossée à la menace constante du doute, puisque la prétention de douter systématiquement de tout ce à propos de quoi un doute peut théoriquement être conçu est purement formelle (comme on l'a vu). Cf. par exemple J. Bouveresse citant C. L. Stevenson pour éclairer l'analyse wittgensteinienne de la certitude et du doute: "C'est une caractéristique typique de l'approche cartésienne que de renverser notre sens moderne de la justice, et de soutenir que toutes nos idées sont coupables jusqu'à ce qu'on ait démontré leur innocence. [...] Beaucoup parmi nous en 
Et Merleau-Ponty identifie explicitement cette façon de «penser le vrai par le faux, le positif par le négatif ${ }^{28} »$ comme la racine d'une opération philosophique générale par laquelle les penseurs ont «cru dépasser les contradictions de la foi perceptive en la mettant en suspens pour dévoiler les motifs qui la soutiennent $\mathrm{t}^{29} »$. Là-contre, à propos de la réalité du monde comme horizon indubitable, Merleau-Ponty parle encore, selon une tonalité bergsonienne toujours saillante, d'une "première positivité dont aucune négation de nos doutes ne saurait être l'équivalent ${ }^{30} »$.

Merleau-Ponty «joue» donc clairement, dans les textes que nous avons en vue, une analyse assez classique (mais " anti-métaphysique ») du néant, du rien, et de la négation contre - notamment - le concept husserlien, «Idéel» et probabiliste, de la réalité, comme concordance idéale des diverses esquisses ou aspects des objets, proposé dans la dernière section des Ideen I (proposition que nous n'avons pas la place de détailler, mais qui finit par redéfinir la « réalité » en termes de justification : la réalité, c'est ce pour quoi nous trouvons des raisons, c'est ce dont l'être fait sens parce qu'il y a des raisons de le poser). Contre Husserl, Merleau-Ponty retrouve l'esprit « bergsonien » qui traverse également (et malgré lui), comme on l'a vu, la réponse d'Austin au "métaphysicien », en montrant que le "problème » moderne du «réalisme » ne prospère que de généraliser indûment, en les décontextualisant, nos $«$ questions ordinaires ${ }^{31}$, comme dit Merleau-Ponty

sont venus à se méfier de cette procédure. Nous avons appris que la démonstration initiale d'innocence a été difficile à trouver. [...] Je ne vois qu'une manière de sortir de cette difficulté - celle qui consiste à laisser tomber complètement l'approche cartésienne et à tenir nos idées pour innocentes jusqu'à ce que leur culpabilité ait été démontrée. Je ne dis pas cela pour proposer un impératif catégorique, mais pour faire une proposition ordinaire - une proposition qui met simplement l'accent en philosophie sur une procédure que nous avons depuis longtemps tenue pour acquise dans la science et dans la vie courante» (C. L. Stevenson, «Some Relations between Philosophy and the Study of Language », Analysis, VIII, 1 (1947-1948), p. 1-9, cité dans J. Bouveresse, Le mythe de l'intériorité. Expérience, signification et langage privé chez Wittgenstein, Paris, Minuit, 1987, p. 601-602).

28. Le visible et l'invisible, op. cit., p. 1673.

29 Ibid., p. 1683.

30. Ibid., p. 1684.

31. Cf. ibid., p. 1733 : « Nos questions ordinaires - "où suis-je ?", “quelle heure est-il ?" -, sont le manque et l'absence provisoire d'un fait ou d'un énoncé positif, trous dans un tissu de choses ou d'indicatifs dont nous sommes sûrs qu'il est continu, puisqu'il y a un temps, un espace, et qu'il ne s'agit que de savoir à quel point de cet espace et de ce temps nous en sommes. La philosophie, à première vue, généralise seulement ce genre de questions. Quand elle demande si l'espace, si le temps, si le mouvement, si le monde existent, le champ de la question est plus ample, mais ce n'est encore, comme la question naturelle, qu'une demi-question, incluse dans une foi fondamentale : il y a quelque chose, et il s'agit seulement de savoir si c'est vraiment cet espace, ce temps, ce mouvement, ce monde, que nous croyons voir ou sentir. La destruction des croyances, le meurtre symbolique des autres et du monde, la coupure de la vision et du visible, de la pensée et de l'être ne nous établissent pas, comme ils le prétendent, dans le négatif ; quand on a ôté tout cela, on s'installe dans ce qui reste [...], et ce qui reste n'est pas rien, ni d'une autre sorte que ce qu'on a retranché ». Nous pensons que relire ce fragment comme la traduction merleau-pontyenne de l'analyse par Austin du « doute ou [de] la question : "est-ce réel ou non ?" ", c'est en clarifier les attendus. 
lui-même quand il critique la généralisation de telles «demi-questions »: «L'interrogation [philosophique] n'est pas un commencement de négation, un peut-être mis à la place de l'être ${ }^{32} »$.

\section{Subversion du point de vue transcendantal}

Et si nous avons voulu détailler un peu cette analyse critique, c'est qu'elle nous paraît être une des pierres fondamentales de l'édifice que construit Merleau-Ponty pour montrer, plus généralement, que le plus grand enseignement de la réduction (au sens de Husserl), c'est l'impossibilité d'une réduction complète, et pour subvertir le thème transcendantal. Comme dit Merleau-Ponty, "la réflexion philosophique a ceci de singulier qu'elle intègre et éclaire la situation à laquelle elle succède, qu'elle se sait comme réflexion sur un irréfléchi, qu'elle éprouve le poids du monde perçu dans sa mise en œuvre même ${ }^{33}$ »; il faut donc opposer à la réduction transcendantale et au thème d'une constitution universelle une nouvelle modalité de penser, à laquelle Merleau-Ponty donne le titre de surréflexion dans le Visible et l'invisible, et qui consiste justement à refuser le geste fondamental de la philosophie réflexive, qui consiste selon lui à ne pouvoir comprendre notre lien natal avec le monde "qu'en le défaisant pour le refaire, qu'en le constituant, en le fabriquant ", en nous replaçant, " en deçà de notre situation de fait, à un centre des choses [...], [en refaisant] en partant de nous un chemin déjà tracé de lui à nous $[\ldots]^{34} \gg$. C'est la critique de ce mouvement «de reprise, de reconquête, de récupération, de retour à soi », de cette " marche à l'adéquation interne », qui autorise à parler chez Merleau-Ponty d'une subversion du geste transcendantal, auquel il propose de substituer ce qu'il appelle

la nécessité d'une autre opération que la conversion réflexive, plus fondamentale qu'elle, d'une sorte de surréflexion qui tiendrait compte aussi d'elle-même et des changements qu'elle introduit dans le spectacle, qui donc ne perdrait pas de vue la chose et la perception brutes, et qui enfin ne les effacerait pas, ne couperait pas, par une hypothèse d'inexistence, les liens organiques de la perception et de la chose perçue, et se donnerait au contraire pour tâche de les penser, de réfléchir sur la transcendance du monde comme transcendance ${ }^{35}$.

Il faut donc fragiliser le criticisme en l'achevant, c'est-à-dire en régressant du transcendantal au monde, au nom de la secondarité essentielle du sujet constituant, par laquelle l'irréfléchi est le présupposé constant de la réflexion $^{36}$ - par où l'on pourrait montrer que partant d'un diagnostic

32. Ibid., p. 1640.

33. Ibid., p. 1670.

34. Ibid., p. 1666-1668.

35. Ibid., p. 1671-1672.

36. Cf. aussi Phénoménologie de la perception, Paris, Gallimard, 1945, p. 419, pour la découverte du « véritable transcendantal, qui n'est pas l'ensemble des opérations constitutives 
analogue sur le criticisme, la phénoménologie de la perception de Merleau-Ponty est l'anti-phénoménologie de l'esprit d'Hegel ${ }^{37}$.

Soulignons, à ce stade, qu'une telle critique de la réduction phénoménologique, qui met en évidence son essentielle circularité, nous semble nouée au refus général, par Merleau-Ponty, du thème du transcendantal - compris de façon critique comme un "pas de côté », un mouvement de retrait, de désengagement, un point de vue «de nulle part », de « côté », de « derrière » ${ }^{38}$, de « surplomb » ou de « survol » (from sideways on, point de vue "non frontal», comme dirait John McDowell, qui parle aussi de « la perspective de l'exil cosmique $\left.{ }^{39} »\right)$. Ce point de vue ou cette posture philosophique dont Merleau-Ponty contribue selon nous à miner les fondements, c'est en un mot celui du pas encore - consistant par exemple à lire une capacité, un phénomène, voire la réalité dans son ensemble, par la négative, d'un point de vue extérieur à eux, en se situant en retrait par rapport à eux, en un point où ils ne sont pas, depuis le lieu fictif ou hypothétique de leur absence - comme si un tel suspens ou un tel détachement, en réalité toujours artificiels, étaient en l'occurrence pensables - et comme s'il fallait à tout prix faire tenir l'expérience par autre chose qu'elle-même.

Ce qui est en question, c'est donc une certaine mythologie dualiste inhérente au projet même d'une fondation transcendantale, qui consiste à placer l'esprit a priori en dehors du monde et à se poser la question angoissée : comment diable va-t-il pouvoir le rattraper ? La critique est alors celle d'un geste qui prospère de postuler une telle séparation radicale de l'esprit et du monde : il s'agit de refuser l'équation même du problème qui consisterait à se demander comment les raccommoder l'un à l'autre. Et il s'agit donc toujours de dégonfler une certaine forme d'inquiétude

par lesquelles un monde transparent, sans ombres et sans opacité, s'étalerait devant un spectateur impartial [...] ». Et ibid., note 1, pour l'analyse des « deux réductions » de l'attitude naturelle, une première fois au bénéfice du monde de la vie, et ultimement au profit d'une subjectivité transcendantale universelle, et pour le refus très clair, de la part de Merleau-Ponty, d'opérer cette seconde réduction: "Husserl dans sa dernière philosophie admet que toute réflexion doit commencer par revenir à la description du monde vécu (Lebenswelt). Mais il ajoute que, par une seconde "réduction", toutes les structures du monde vécu doivent à leur tour être replacées dans le flux transcendantal d'une constitution universelle où toutes les obscurités du monde seraient éclaircies. Il est cependant manifeste que c'est de deux choses l'une : ou bien la constitution rend le monde transparent, et alors on ne voit pas pourquoi la réflexion aurait besoin de passer par le monde vécu, ou bien elle en retient quelque chose et c'est qu'elle ne dépouille jamais le monde de son opacité ».

37. Sur ce point, cf. A. Grandjean, Critique et réflexion. Essai sur le discours kantien, Paris, Vrin, 2009, p. 55-56.

38. Comme l'écrit Wittgenstein à propos des « règles ", " on ne peut pas pénétrer derrière les règles, parce qu'il n'y a pas de derrière » (L. Wittgenstein, Grammaire philosophique, trad. fr. M.-A. Lescourret, Paris, Gallimard, «Folio », 1980, p. 170).

39. Cf. J. McDowell, «Having the World in View : Sellars, Kant and Intentionality », The Journal of Philosophy, vol. 95, 9, 1998, conf. 1, sect. 4 ; ou encore J. McDowell, « Noncognitivisme et règles », trad. fr. J.-P. Narboux, Archives de philosophie, 2001/3, tome 64, p. 457-477 ; p. 467-473). 
philosophique, qui peut être aussi un étonnement feint ${ }^{40}$. C'est la logique même d'un tel étonnement, gouvernant la posture transcendantale ${ }^{41}$ (laquelle consiste par exemple à se demander, éventuellement avec stupeur, comment il est seulement possible que nous ayons accès à quelque chose, disons, comme le monde), qui serait par avance fautive. Ce qui est ainsi critiqué à travers le refus du point de vue dit transcendantal, c'est donc toujours le fait même de poser la question de l'objectivité de nos pensées, ce qui suppose de se placer là où la référence est quelque chose à rechercher, à conquérir, à fonder - avec l'idée qu'est essentielle au point de vue transcendantal une forme de séparation, le réel étant pensé sous le régime, en tout cas, de ce qu'on n'a pas au départ: pour qu'il y ait une déduction transcendantale à faire, il faut qu'on se soit mis dans la position imaginaire où ce n'est pas gagné ${ }^{42}$.

La critique (qui peut s'adresser à toutes les philosophies « d'inspiration transcendantale ») consiste donc toujours, en particulier, à " montrer qu'on aurait tort d'aborder [la question de la prise de la pensée sur le monde] par la négative en demandant en quoi consisterait le "fait", pour la pensée comme telle, de ne pas ou de ne plus avoir prise sur le monde (de ne pas ou de ne plus être ne serait-ce que capable d'être vraie ou fausse), pour ensuite spécifier les conditions en vertu desquelles la pensée surmonte toujours déjà une telle éventualité, avant même d'être vraie ou fausse ». Il s'agit donc de montrer que «la question d'une telle éventualité appelle bien moins

40. Pour une problématisation de cet «émerveillement/inquiétude feint(e)», à ne pas confondre avec l'" admiration du monde » des Anciens, cf. H. Blumenberg, Le souci traverse le fleuve, trad. fr. O. Mannoni, Paris, L'Arche, 1990, p. 67-68.

41. C. Travis qualifie précisément de «transcendantale» toute démarche philosophique consistant à prétendre se situer en dehors des pratiques (notamment linguistiques), de nos formes de vie et de leurs critères, pour pouvoir en juger de l'extérieur. Cf. notamment C. Travis, Les liaisons ordinaires: Wittgenstein sur la pensée et le monde, trad. fr. B. Ambroise, Paris, Vrin, 2003.

42. Ce schème critique est au cœur du «réalisme» de Jocelyn Benoist. Cf. par exemple, pour une formule particulièrement frappante appliquée à la question de la "réalité du social », J. Benoist, Éléments de philosophie réaliste, Paris, Vrin, 2011, p. 166-168 : «[L]a philosophie moderne se caractérise par l'existence d'un "problème du social". C'est en rompant les fils de l'existence sociale qu'elle prétend la rendre visible, y compris lorsque, en un second temps, elle feint de la redécouvrir au plus près de nous, comme s'il y avait là une surprise. Mais c'est oublier que les concepts mêmes dont elle est partie, formulant ses questions, sur la réalité du social et sur le partage entre ce qui est social et ce qui ne l'est pas, renvoyaient à des aspects de cette réalité sociale même. Il n'y a pas d'exil asocial depuis lequel la question de "la société" puisse être posée et la "possibilité d'une île", en son sens transcendantal, est une fausse question. Tout au plus y a-t-il une prise de conscience possible de notre être social, qui dissout certaines perplexités conceptuelles. Si nous n'avons pas le social, personne, pas même le tiers, ne nous le donnera. Mais nous l'avons, et c'est depuis cette réalité première que nous tombons parfois dans cet embarras étrange de nous demander si nous l'avons. [...] La réalité sociale, comme la réalité en général, ne peut pas manquer c'est le genre de concept auquel on ne voit pas selon quel sens la notion de "manque" pourrait s'appliquer - mais nous pouvons très bien y manquer - c'est-à-dire ne pas être en mesure de nous reconnaître comme y étant. Et sans doute est-ce ce qui donne quelque motif, à la limite du non-sens, de l'appeler une "réalité" ». 
une solution qu'une dissolution [...] ; de montrer que le fossé "logique" qui semble naître sous nos pieds lorsque nous imaginons que la pensée n'ait pas ou plus prise sur le monde n'est que l'illusion d'un fossé, et que nous n'imaginons pas ce que nous croyons alors imaginer $\gg{ }^{43}$.

\section{L'apagogie radicalisée}

Arrêtons-nous, pour finir, sur ce point, qui mérite une clarification conceptuelle, afin de mieux saisir l'esprit du geste philosophique que nous tentons de décrire. En effet, nous pourrions très bien avoir tendance, à l'inverse, à enrôler ce genre d'inspiration philosophique sous la bannière transcendantale - ce n'est pas un hasard, d'ailleurs, si le genre de critique que nous avons en vue fournit très exactement son principe à ce que James Conant appelle «le traitement kantien du scepticisme » ${ }^{44}$. C'est que, en un sens, ce que montre Kant dans la Critique de la raison pure, c'est que si la pensée a prise sur le monde, c'est que le monde et la pensée sont toujours déjà (le syntagme est important) à la mesure l'un de l'autre, qu'ils sont nécessairement en harmonie l'un avec l'autre, et que les menaces idéaliste ou sceptique peuvent être dissoutes par l'absurde, en tant qu'elles ne sont que des «parodies » de menaces. Au fond, l'esprit du transcendantal consisterait alors, précisément, à exhiber ceci que c'est une seule et même chose pour une pensée d'avoir un contenu, et d'avoir prise sur le monde, d'avoir ce qu'on peut appeler une "validité objective ». Il devient alors possible d'inscrire le genre de gestes critiques que j'ai envisagés dans l'héritage même de la démarche transcendantale, autour de l'idée, comme dit, justement, Strawson pour résumer l'esprit de ce qu'il appelle « argument transcendantal », à la dernière page de The Bounds of Sense, que "nous n'avons pas les mots pour dire ce que ce serait sans eux ${ }^{45} »$.

Autrement dit, la difficulté ou l'ambiguïté que nous tentons de cerner résident dans le fait que le genre de méthode philosophique dont nous essayons de dégager l'esprit idéal-typique - celle de Wittgenstein par exemple, mais aussi bien, en un sens particulier, celle de Merleau-Ponty peut à la fois être érigée en exemplaire dissolution du geste transcendantal, et en même temps peut être elle-même, en un sens, rangée sous la bannière transcendantale. En effet, la frontière n'est parfois pas nette, pas étanche, pas claire, entre d'une part la revendication et d'autre part le refus du

43 J.-P. Narboux, «Y a-t-il jamais de surprise en logique ? Logique et téléologie : Kant et Wittgenstein ", in L'année 1790. Critique de la faculté de juger, C. Bouton, F. Brugère et C. Lavaud (dir.), Paris, Vrin, 2008, p. 283.

44. Cf. J. Conant, «Two Varieties of Skepticism», in Rethinking Epistemology, vol. 2, G. Abel and J. Conant (dir.), Berlin, Walter de Gruyter, 2012, p. 1-73.

45. Cf. P. F. Strawson, The Bounds of Sense. An Essay on Kant's Critique of Pure Reason, Londres, Methuen, 1966. Sur cette question, cf. aussi S. Laugier, "Langage, scepticisme et argument transcendantal », Cahiers de philosophie de l'Université de Caen, n 35, 2000. 
transcendantal, notamment lorsqu'il s'agit de développer des stratégies argumentatives pour contrer le scepticisme ou réfuter l'idéalisme - et cette ambiguïté transparaît nettement dans la prose même de Merleau-Ponty. La différence n'est pas nécessairement claire, donc, entre deux stratégies : d'un côté, une stratégie anti-transcendantale et en un certain sens naturaliste, qui montre par exemple que le sceptique fait fond sur une possibilité alléguée mais fictive de détachement vis-à-vis de la réalité, à laquelle il faut opposer un principe d'immanence radicale sans chercher à réfuter le sceptique sur son terrain, celui d'un gouffre qu'il faudrait franchir entre l'esprit et le monde ${ }^{46}$; de l'autre côté, une stratégie qu'on pourrait dire cette fois transcendantale, au sens de ce que Strawson appelle précisément un "argument» transcendantal, lequel prend au contraire au sérieux la possibilité d'un tel détachement ou d'une telle vue de côté, mais précisément pour montrer qu'elle s'autodétruit par réduction à l'absurde et autocontradiction. La différence n'est pas claire, autrement dit, entre dissoudre un problème en en acceptant la formulation philosophique classique, d'un côté, et, de l'autre, déjouer jusqu'au problème lui-même ${ }^{47}$.

Pourquoi cette ambiguïté de la référence, positive ou négative, au motif «transcendantal»? Sans doute parce que le «transcendantal» n'est pas seulement un thème général, mais aussi un style argumentatif: un « argument transcendantal », selon la reformulation proposée par Strawson de ce concept, consiste toujours à montrer - donc en un sens par l'absurdeque si l'on n'accepte pas tel ou tel concept (celui de cause par exemple), on ne peut penser ni agir comme nous le faisons, puisque ces concepts ou séries de concepts sont impliqués nécessairement et toujours déjà présupposés dans les opérations cognitives que de fait, de toute façon, nous réalisons et ne pouvons pas ne pas réaliser. Donc, cette fois : le transcendantal comme « argument », et comme argument négatif, qui consiste toujours à objecter à l'adversaire qu'il ne peut pas penser ce qu'il croit penser, parce que le transcendantal, par-delà toutes les questions et tous les doutes imaginables, est-ce sans quoi ce qui est effectif ne serait pas possible, donc ce qu'on admet toujours déjà même en tentant de le réfuter ${ }^{48}$.

46. Avec l'idée que le sceptique, ou l'idéaliste, et celui qui tente de les réfuter, partagent au fond les mêmes prémisses erronées - stratégie argumentative de triangulation, d'ascendance wittgensteinienne, popularisée notamment par H. Putnam et par les partisans d'une interprétation « austère » du non-sens, dont le principe serait le suivant : tenter de démontrer ou de justifier le réalisme, ce serait toujours déjà tomber dans le piège tendu par les adversaires du réalisme en acceptant ce que J. Benoist appellerait la problématique de «l'accès », celle d'un gouffre qu'il faudrait franchir entre l'esprit et le monde.

47. Cf., en l'occurrence à propos de Wittgenstein, É. Marrou, « La critique de la factualité du jugement : le problème de l'induction à l'épreuve dans De la certitude », Klésis, 9, 2008, p. 107: "Le cœur de la difficulté est donc ici de savoir s'il s'agit seulement pour Wittgenstein de dissoudre le problème en en acceptant la formulation philosophique classique, ou s'il déjoue jusqu'au problème lui-même ».

48. Ce qui justifie d'ailleurs, par exemple, l'usage du syntagme «transcendantal» pour définir le type d'argument ou de réfutation mis en œuvre par Aristote en Métaphysique Gamma contre celui qui refuse le principe de non-contradiction (et qui peut déjà s'apparenter 
Le thème transcendantal devient alors beaucoup plus compatible avec les perspectives apparemment anti-transcendantales que nous avons pu évoquer - en l'occurrence en insistant sur Merleau-Ponty. Et l'idée serait alors la suivante, pour embrouiller encore l'écheveau: la critique du transcendantal telle que nous l'avons esquissée serait peut-être au fond, en un sens, le dernier mot ou dernier tour du transcendantal lui-même, au sens où nous aurions peut-être affaire là encore, avec ce type de perspectives, à la tentation de découvrir « l'argument transcendantal qui mettrait fin à tous les arguments transcendantaux ${ }^{49} »$. Et la critique du thème transcendantal peut alors elle-même être dite transcendantale, mais cette fois dans sa méthode, dans son mode de raisonnement ou de probation - au sens où elle revient à dire, d'une façon ou d'une autre : " nous ne pouvons pas faire autrement », «nous y sommes toujours déjà » (et il n'y a là aucun abîme à franchir, par exemple entre l'esprit et le monde) - formule qu'il est intéressant de rapprocher de la formule logique récurrente du discours kantien, et en un sens caractéristique de la perspective transcendantale, qui est celle du «pas sans » ou du «impossible sans » ${ }^{50}$. La forme ramassée d'une telle perspective, telle qu'elle est explicitée dans la « Doctrine transcendantale de la méthode » de la Discipline de la raison pure ${ }^{51}$, c'est celle de ce que les logiciens appellent le modus tollens de la preuve apagogique, ou raisonnement par l'absurde, par opposition à la preuve «directe ou ostensive » qui part d'un principe et montre qu'une conséquence en découle nécessairement. La formule du raisonnement par l'absurde est en effet la suivante : si nonP, alors nonQ, or $\mathrm{Q}$, donc $\mathrm{P}$; soit : [(nonP $\rightarrow$ nonQ) $\cap \mathrm{Q}] \rightarrow \mathrm{P}$. Autrement dit : on conclut de la fausseté de la conséquence à la fausseté du principe et donc à la vérité de la proposition contradictoire; ou, comme dit Kant dans la Logique: «si je conclus la vérité d'une proposition de la fausseté de l'opposé, j'en produis une preuve apagogique ${ }^{52} »$. Et, par exemple: si le principe de causalité n'avait pas de valeur objective, alors la détermination d'un événement comme objet de l'expérience, et donc l'expérience elle-même, seraient impossibles ; or la détermination de

à la forme canonique du raisonnement par l'absurde). Cf. B. Cassin, «Parle, si tu es un homme ", in La Décision du sens. Le livre Gamma de la Métaphysique d'Aristote, introduction, texte, traduction et commentaire, B. Cassin et M. Narcy (dir.), Paris, Vrin, 1989.

49. R. Rorty, "Transcendantal Arguments, Self-reference and Pragmatism », in P. Bieri, R. P. Hortsman et L. Kruger (dir.), Transcendental Arguments and Science, Dordrecht, D. Reidel, 1979, p. 78.

50. D. Henrich, «Kant's Notion of a Deduction and the Methodological Background of the first Critique », in Kant's transcendental deductions, E. Förster (dir.), Stanford, Stanford University Press, 1989, p. 44. Pour une étude approfondie de ce thème et de ce problème, cf. A. Grandjean, Critique et réflexion, op. cit., p. 139 et suiv., qui insiste, précisément, sur la structure argumentative spécifique du discours transcendantal, et propose une détermination particulièrement claire de la spécificité kantienne du thème transcendantal et du style philosophique qui lui est inhérent, en démontrant en particulier son caractère négatif, régressif et présupposant.

51. Cf., par exemple, E. Kant, Critique de la raison pure, A782/B810 ; A788-789/B816-817.

52. E. Kant, Logique, Introduction, IX, Ak. IX, 71. 
l'expérience est un fait, de sorte que le principe de causalité a une valeur objective ; donc : pas d'expérience possible sans la catégorie de causalité. Et ce thème du « impossible sans », structure essentielle du discours kantien (tel que A. Grandjean notamment en restitue le principe), peut alors, en quelque sorte, retrouver des couleurs, si l'on accepte la légitimité et la fécondité d'un mode spécifique de justification - qu'on pourra dire transcendantale - dont le propre serait de faire fond sur l'impossibilité d'imaginer autre chose, sur l'impossibilité de dire ce à quoi un supposé monde (ou expérience, ou concept, ou faculté) alternatif ressemblerait. Un tel mode de justification dont on voudrait suggérer qu'il irrigue en profondeur des pensées aussi différentes que celles, disons, de Merleau-Ponty ou de Wittgenstein - revient donc à actionner le levier de ce que nous " présupposons » toujours déjà, y compris à notre insu, afin de réduire à l'absurde et à l'autocontradiction la thèse opposée, qu'elle soit sceptique, idéaliste, relativiste, ou autre. Il s'agit là, donc, d'une forme très particulière d' « apagogie » ou de raisonnement par l'absurde, dans lequel ce qui est pointé en un sens, c'est l'impossibilité pure et simple de parcourir la proposition contradictoire à celle qu'on entend établir, fût-ce fictivement et pour en démontrer l'absurdité. Et un tel geste (dont une formule possible serait bien: refus de penser le positif par le négatif) peut bel et bien revendiquer une ascendance kantienne, puisque la preuve des propositions transcendantales, chez Kant, passe toujours par «l'établissement de l'impossibilité de l'expérience comme conséquence de la négation de leur vérité, impossibilité qui contrevient à chaque fois à ce que Kant appelle la "présupposition ${ }^{53}$ ", parce que l'expérience est un fait ${ }^{54}{ }^{\prime}$.

Et c'est exactement le même genre d' « apagogie radicalisée » que nous pensons pouvoir retrouver par exemple chez Wittgenstein, lorsque celui-ci met en scène les chimères sceptiques des philosophes pour les vider de sens et en faire apparaître le caractère superflu: nous pourrions montrer que l'originalité des fictions ou expériences de pensée wittgensteiniennes ${ }^{55}$ est qu'elles sont opératoires précisément en tant qu'elles s'avèrent ne pas en être vraiment, c'est-à-dire en tant qu'elles «tournent court» et servent à dissoudre la possibilité même de se placer en extériorité par rapport à

\section{E. Kant, Critique de la raison pure, A737/B765.}

54. A. Grandjean, Critique et réflexion, op. cit., p. 138.

55. Cf., par exemple, l'expérience de pensée des « vendeurs de bois », c'est-à-dire la version wittgensteinienne du spectre ou «scénario » frégéen d'une pensée logiquement étrangère ou d'une «folie» logique (d'un logically alien mind), scénario dissous en même temps qu'énoncé - le spectre d'une autre logique étant d'un même mouvement mis en scène et exhibé comme inconsistant. Cf. L. Wittgenstein, Remarques sur les fondements des mathématiques, trad. fr. M.-A. Lescourret, Paris, Gallimard, 1983, p. 207 et suiv. Nous suivons, pour l'interprétation des expériences de pensée wittgensteiniennes, l'esprit du commentaire de É. Marrou (cf. notamment É. Marrou, « Austin et Wittgenstein : le problème des autres esprits à l'épreuve ", in Cahiers de philosophie du langage 7. Wittgenstein en confrontation, D. Perrin et L. Soutif (dir.), vol. 7, Paris, L'Harmattan, 2011, p. 111 et suiv.). Pour une problématisation plus générale de cette question du statut de l'" other-mindedness », cf. J. Lear, "Leaving the World Alone », in The Journal of Philosophy, 79(7), (1982), notamment p. 386-389. 
notre pensée, en la considérant indépendamment des résultats qu'elle donne effectivement. L'invention de jeux de langage, les variations fictives autour de nos concepts, l'invocation de tribus imaginaires, la mise en scène des arguments sceptiques dans des saynètes, ces procédés wittgensteiniens consistent toujours non pas à exhiber ou supposer une extériorité ou une altérité véritables, mais à nous confronter aux limites de ce que nous pouvons imaginer en réduisant à l'absurde les suppositions sceptiques, ainsi retournées contre elles-mêmes. Le principe argumentatif est bien celui dont nous essayons de cerner les contours : l'idée est qu'on ne peut pas même s'installer dans la possibilité ou l'hypothèse contrefactuelle brandie par le sceptique, puisque la négation qui sert de prémisse à la preuve ne peut recevoir aucun sens.

On mesure donc en tout cas, au passage, l'ambiguïté du rapport de cette constellation théorique, dont nous avons voulu présenter l'un des modes argumentatifs, avec le thème transcendantal et avec Kant, puisque cette constellation qui apparemment prospère sur la critique de la "posture » transcendantale, peut aussi bien être lue comme une certaine forme, peut-être hétérodoxe, de postérité kantienne. Et d'ailleurs Merleau-Ponty lui-même ne s'y trompait pas, comme le montrent les précisions suivantes, qui accompagnent sa thématisation de la «surréflexion» évoquée plus haut: "Lorsque Kant justifie chaque démarche de son Analytique par le fameux : "si un monde doit être possible", il souligne que son fil directeur lui est donné par l'image irréfléchie du monde, que la nécessité des démarches réflexives est suspendue à l'hypothèse "monde" et que la pensée du monde que l'Analytique est chargée de dévoiler n'est pas tant le fondement que l'expression seconde du fait qu'il y a eu pour moi expérience d'un monde, qu'en d'autres termes la possibilité intrinsèque du monde comme pensée repose sur le fait que je peux voir le monde, c'est-à-dire sur une possibilité d'un tout autre type ${ }^{56}$ ». C'est là en même temps reconnaître que le discours transcendantal trouve son originalité même dans un mode de probation et une structure argumentative originaux, qui vont de pair avec une déliaison typiquement kantienne et anti-métaphysique de la nécessité et de la fondation, et qui permettent au contraire d'enrôler Kant dans le camp de «l'esprit réaliste» dont nous tentons de cerner les motifs ${ }^{57}$. Il est en tout cas

56. M. Merleau-Ponty, Le visible et l'invisible, op. cit., p. 1668.

57. Il existe d'ailleurs une réflexion très précise de Kant lui-même, par exemple dans une lettre à Herz de mai 1789 (E. Kant, Lettre à Herz du 26 mai 1789, Ak. XI, 51), sur les limites de la question «pourquoi ? » discutée plus haut, réflexion par laquelle Kant relativise l'usage du principe de raison : l'inconditionné en effet, loin d'être fondé par soi, est positivement sans raison, infondé, et commande justement l'élision de la question «pourquoi ? ». Sur ce point, cf. A. Grandjean, Critique et réflexion, op. cit., p. 181 : « [Chez Kant], répondre aux questions «pourquoi y a-t-il le transcendantal plutôt que rien? » et «pourquoi le transcendantal est-il ainsi plutôt qu'autrement? » exigerait que du transcendantal on puisse faire un objet qu'il s'agirait de mesurer depuis son dehors, étalon qui en ferait une variable en rendant la finitude contingente à ses propres yeux. Or la finitude n'est finitude que de ne pouvoir se mettre à distance d'elle-même. La raison finie ni ne peut répondre de son propre pourquoi, ni n'est 
possible à la fois, dans le travail même de ces ambiguïtés, de critiquer le motif argumentatif consistant à " penser par la négative», et, d'un autre point de vue, de lui redonner quelques couleurs, à travers une défense de l'usage philosophique de l'apagogie (ou raisonnement par l'absurde).

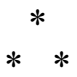

Voici en tout cas ce qui constituait l'horizon de notre lecture de Merleau-Ponty armée d'un point de vue austinien : la critique de ce geste récurrent consistant à transférer sur un terrain universel et décontextualisé un usage normatif et local de la notion de réalité (geste présent par excellence au principe de la question "Pourquoi y a-t-il quelque chose plutôt que rien? ») - geste qui consiste aussi notamment (il faudrait le montrer en détail) à faire de phénomènes bien particuliers, qui ont une contextualité très forte (illusion, hallucination, correction) l'équation du problème en général (alors même que ledit phénomène n'est possible que parce que la perception fonctionne dans des registres beaucoup plus basaux, selon une «présence si forte, comme dit J. Benoist, qu'elle leste toutes ces formes d'absence par lesquelles on se donne du jeu et circule à sa surface $\left.{ }^{58} \gg\right)$. Merleau-Ponty (ou $\mathrm{du}$ moins certains aspects et passages de son œuvre) s'avère donc être un représentant exemplaire de cette position critique générique qui refuse la «vue de côté» ou de «survol» et les «questions » généralisantes, hypostasiées et décontextualisées, des métaphysiciens, tout en exploitant la fécondité de cette forme logique du «impossible sans » pour réduire à l'absurde lesdites « fausses questions ».

C'est pourquoi nous pensons qu'il est possible d'inscrire son œuvre dans une certaine histoire philosophique contemporaine que l'on pourrait rassembler, par-delà la différence évidente des contextes théoriques sous lesquels elle peut se décliner, sous le titre d'un «esprit réaliste» en philosophie, c'est-à-dire d'une attention ou d'une disponibilité descriptive au réel qui rompt avec l'habitude consistant à exiger de lui qu'il «montre patte blanche » en répondant à des défis insensés tels que celui du doute hyperbolique, ou à des alternatives fallacieuses comme celle du «quelque chose » et du «rien» imposée par ledit "problème ontologique ». Et cet « esprit réaliste» consiste bien en un sens, pour le dire de façon ramassée, à «détranscendantaliser» la pensée, c'est-à-dire à ôter toute pertinence à la question de savoir si on a ou pas le réel, et donc à la question transcendantale ainsi redéfinie: comment un accès à la réalité est-il seulement possible?

justifiée à le demander »; et le transcendantal, chez Kant lui-même, est au fond positivement sans raison et sans pourquoi.

58. Cf. J. Benoist, Concepts. Introduction à l'analyse, Paris, Cerf, 2010, p. 48. 